\title{
Pre-Conceptual Design Activities for the Materials Plasma Exposure Experiment
}

\author{
Arnold Lumsdaine, Juergen Rapp, Venugopal Varma, Thomas Bjorholm, Craig Bradley, John \\ Caughman, Robert Duckworth, Richard Goulding, Van Graves, Dominic Giuliano, Timothy Lessard, \\ Dean McGinnis, and Steven Meitner
}

Oak Ridge National Laboratory, Oak Ridge, Tennessee

\begin{abstract}
The development of next step fusion facilities such as DEMO or a Fusion Nuclear Science Facility (FNSF) requires first closing technology gaps in some critical areas. Understanding the material-plasma interface is necessary to enable the development of divertors for long-pulse plasma facilities. A pre-conceptual design for a proposed steady-state linear plasma device, the Materials Plasma Exposure Experiment (MPEX), is underway. A helicon plasma source along with ion cyclotron and electron Bernstein wave heating systems will produce ITER divertor relevant plasma conditions with steady-state parallel heat fluxes of up to $40 \mathrm{MW} / \mathrm{m}^{2}$ with ion fluxes up to $10^{24} / \mathrm{m}^{2}$-s on target. Current plans are for the device to use superconducting magnets to produce 1-2 Tesla fields. As a steady-state device, active cooling will be required for components that interact with the plasma (targets, limiters, etc.), as well as for other plasma facing components (transport regions, vacuum tanks, diagnostic ports). Design concepts for the vacuum system, the cooling system, and the plasma heating systems have been completed. The device will include the capability for handling samples that have been neutron irradiated in order to consider the multivariate effects of neutrons, plasma, and high heat-flux on the microstructure of divertor candidate materials. A vacuum cask, which can be disconnected from the high field environment in order to perform in-vacuo diagnosis of the surface evolution is also planned for the facility.
\end{abstract}

Keywords: plasma facing components; plasma-material interactions; R\&D facilities; linear plasma experiments.

\section{Introduction}

The development of long-pulse nuclear fusion devices such as ITER, FNSF and DEMO depends on long operating lifetimes of plasma facing components in their divertors [1-3]. The development of those plasma facing components and materials requires facilities for testing them at reactor relevant conditions. This includes relevant divertor plasma parameters ( $\mathrm{n}_{\mathrm{e}}$ up to $\sim 10^{21} \mathrm{~m}^{-3}$, $\mathrm{T}_{\mathrm{e}}=1-15 \mathrm{eV}$ ), plasma fluxes of up to $10^{24} \mathrm{~m}^{-2} \mathrm{~s}^{-1}$, lifetime relevant fluence of $\sim 10^{30}-10^{31}$ ions $/ \mathrm{m}^{2}$, high PFC ambient temperature, and relevant displacement damage as a result of neutron irradiation [4]. Developing the science of plasma-material interactions and the technology of plasma facing material components will require new facilities. Development of next-stage plasma facing materials must be underpinned by a fundamental understanding of how prototypical plasmas interact with surfaces. So new experimental facilities must be built that are capable of carrying out this research [5-6].

The Material-Plasma Exposure eXperiment (MPEX), a superconducting magnet, steady state device, is

\footnotetext{
This manuscript has been authored by UT-Battelle, LLC under Contract No. DE-AC05-00OR22725 with the U.S. Department of Energy. The United States Government retains and the publisher, by accepting the article for publication, acknowledges that the United States Government retains a non-exclusive, paid-up, irrevocable, world-wide license to publish or reproduce the published form of this manuscript, or allow others to do so, for United States Government purposes. The Department of Energy will provide public access to these results of federally sponsored research in accordance with the DOE Public Access Plan (http://energy.gov/downloads/doe-publicaccess-plan)

lumsdainea@ornl.gov
}

proposed to address the above mentioned conditions. A pre-conceptual design of the device is shown in Fig. 1. This facility will have the unique feature of being able to conduct accelerated lifetime tests of plasma facing components. As an example, for tungsten the net erosion yield for divertor plasmas with $T_{e}=10-15 \mathrm{eV}$ has to be reduced to $10^{-6}$ by effective re-deposition processes to achieve the required lifetime. MPEX will utilize a new high-intensity plasma source concept based on RF technology. This source concept will allow the experiment to cover the whole range of expected plasma conditions in the divertor of a future fusion reactor, including the very high densities [7]. It will be able to study erosion and re-deposition in correct geometries with relevant electric and magnetic fields in-front of the target. The source system will consist of a helicon antenna for high density plasma production. This plasma will be subsequently heated by Electron Bernstein Waves and Ion Cyclotron Resonance heating. The total heating power will be up to $800 \mathrm{~kW}$. The device is sized based on extensive plasma-neutral modeling with state-of-the-art codes, which are also used for the design of the ITER divertor and the W7-X divertor [7]. The plasma production and heating schemes have been modeled as well, which led to the definition of the magnetic field profile. The facility is being designed to be compatible with a later upgrade to handle neutron irradiated samples. In particular, the device is designed to allow for an impurity contamination control with docking station concepts. 


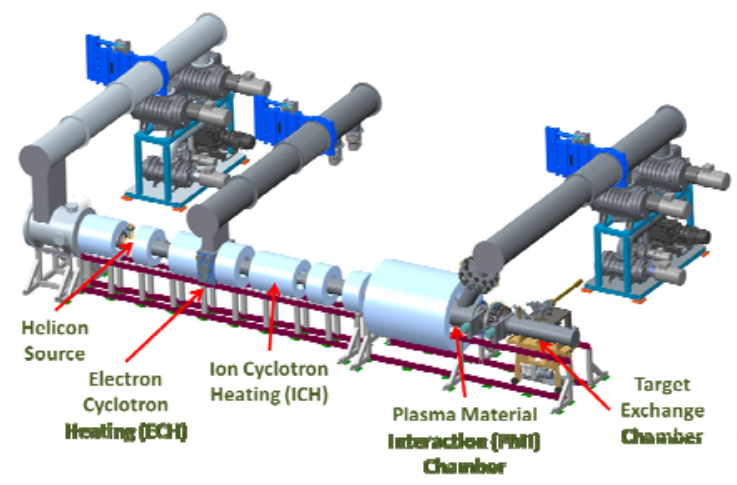

Fig. 1. Pre-conceptual model of MPEX.

\section{Design activities}

The design of MPEX can be conceptually broken up into several activities:

- Plasma sources and heating - helicon, electron cyclotron heating $(\mathrm{ECH})$ and ion cyclotron heating (ICH);

- Magnet and cryo system;

- Vacuum system and components;

- Plasma material interaction (PMI) chamber;

- Water cooling system and components.

The status of the pre-conceptual design activities for each of these systems is detailed below.

\subsection{Plasma source and heating}

The plasma source uses a dual half turn helical antenna (Fig. 2). Power is coupled into the plasma through the antenna at a frequency of $13.56 \mathrm{MHz}$. The antenna is located in air, and the power is coupled through a cylinder forming the vacuum boundary in this region, referred to here as a "window". The antenna is located outside the vacuum due to the fact that high neutral pressures in the range $0.1-3 \mathrm{~Pa}$ are required in the helicon section in order to produce the required plasma densities, and at this pressure and power level antenna sputtering would otherwise be likely to occur that could contaminate material samples being tested. However, a drawback is that up to $20 \%$ of the power launched by the antenna can be deposited on the inner surface of the window due to RF-plasma sheath interactions and the production of hot neutrals. The window thus must be adequately cooled so that thermal stresses do not become excessive. The composition of the window and the design of the water cooling path are areas of current R\&D. The inner window must have a low dielectric constant, but high thermal conductivity to reduce thermal stresses. Aluminum nitride has been used for a prototype window. Alumina is also among other materials under consideration. A less conductive material such as quartz can be considered for the outer window (as shown in Fig. 2) as the thermal conductivity is not as important because the thermal loading will not be significant.

Electron Bernstein Wave heating with $200 \mathrm{~kW}$ of 28 $\mathrm{GHz}$ gyrotron power is planned to for the electron heating scheme, since the ordinary electromagnetic waves in the electron cyclotron range will not propagate in the envisioned density range and magnetic field range planned in MPEX. Electrostatic electron Bernstein waves (EBW) propagate in those overdense plasmas in which the plasma frequency is larger than the electron cyclotron frequency. The ECH power will be transmitted to MPEX through waveguide to a radio frequency (RF) "box" (Fig. 3). This box will contain an actively cooled, steerable mirror to reflect the microwaves to the resonant zone. The ECH region is required to be at a low pressure (on the order of 0.01Pa).
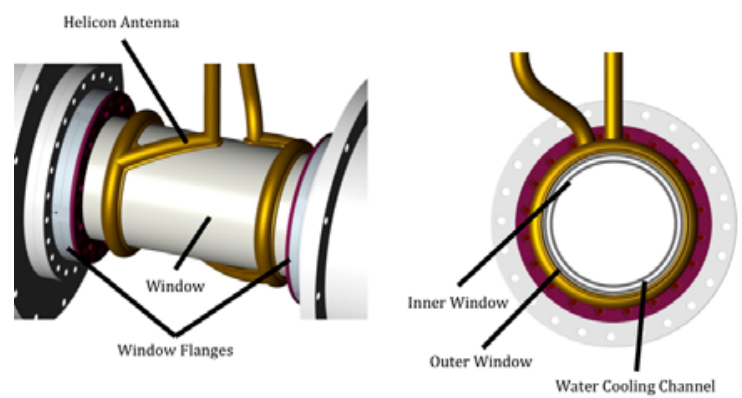

Fig. 2. Helicon antenna and window.

The ICH power will be coupled through two modified antennas with half-turn helical twists, similar to the helicon antenna, but with the opposite helicity (Fig. 3). These antennas will also be outside of the vacuum, but, unlike the helicon, must launch from an area of high field $(1.8 \mathrm{~T})$, and so will be inside one of the superconducting magnets.

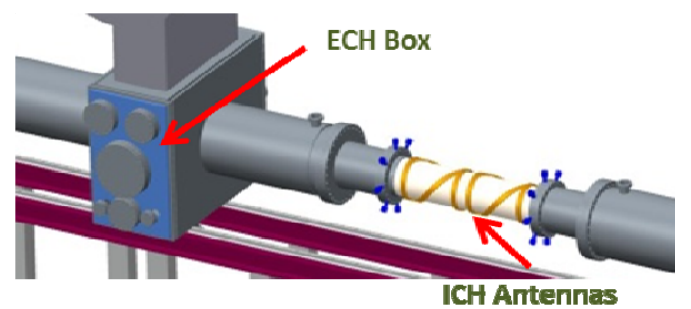

Fig. 3. EC and IC heating regions (magnets not shown).

\subsection{Magnet system}

The magnet system for MPEX consists of five systems (Helicon, ECH, ICH, Transport, \& Target) that can be operated independently, but work together to produce a plasma density at the target area that is beneficial for accelerated testing of plasma materials for fusion environments. Fig. 4 shows the layout and relative size of the magnet system relative to the different components of MPEX. Given the desire to operate the system steady-state, low temperature superconducting magnets will be used. This selection was driven by the maturity of the conductor technology as well as other supporting technology relative to other alternatives 
currently commercially available. Different regions of MPEX have different requirements for magnetic field strength. The ratio of the on-axis field at the helicon magnet to the lowest field in the helicon should be $>3$. The field in the resonant zone of the ECH box should be $>1.0 \mathrm{~T}$. The ICH magnets should produce a field of 1.8 $\mathrm{T}$, dropping to $1.6 \mathrm{~T}$ downstream of the antenna. The on-axis field in the target region should be $>1.0 \mathrm{~T}$. Because of the size of the PMI chamber, and the space needed for diagnostic, the cryostat of the target magnets is required to have an inner bore of $1.15 \mathrm{~m}$.

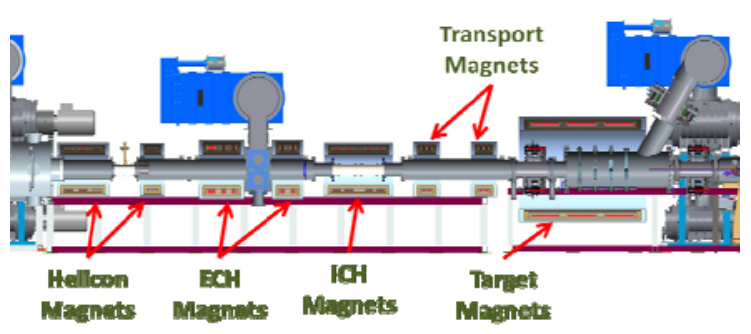

Fig. 4. Cross-section of magnet systems.

\subsection{Vacuum systems}

The vacuum system is designed to achieve conditions similar to the ITER divertor at the MPEX target (see Fig. 1 and Fig. 4). The pressure in the PMI chamber is required to be 1-10 $\mathrm{Pa}$. In order to achieve the lower pressure in the ECH box ( $\sim 01 \mathrm{~Pa})$, skimmers will be placed in several regions of the vessel to prevent the transport of neutral particles. It is foreseen to utilize large roots blower trains at the PMI chamber and the dump tank, each with a pumping speed of 22,500 $\mathrm{m}^{3} / \mathrm{hr}$. This will keep the pressure below $10 \mathrm{~Pa}$ even with Deuterium at the highest gas flow $(7 \mathrm{slm})$. The ECH chamber, which requires a lower pressure, will be pumped by two parallel turbo pumps with a pumping velocity of $2300 \mathrm{l} / \mathrm{s}$ each.

\subsection{PMI chamber}

The PMI chamber of MPEX is where the target will be exposed to plasma and where a large diagnostic suite for in-situ plasma and PMI measurements will be made (Fig. 5). The chamber is designed with 48 diagnostic ports, some with a $63 \mathrm{~mm}$ flange and some with a 150 mm flange. The chamber, and all diagnostic ports (as well as any diagnostic that will be plasma facing) will be water cooled. The vessel will have an inner diameter of $500 \mathrm{~mm}$, with a radial clearance of at least $300 \mathrm{~mm}$ between the outer diameter of the vessel and the inner bore of the magnet cryostat in order to allow for diagnostic access. In order to allow the target chamber to be moved while maintaining vacuum, and to prevent the need for close human interaction after testing with irradiated targets, an automated vacuum coupling system has been designed that can operate hydraulically (Fig. 6). The hydraulic system is capable of providing enough force to compress an annealed copper gasket, with knifeedge seal on a DN250 vacuum flange.

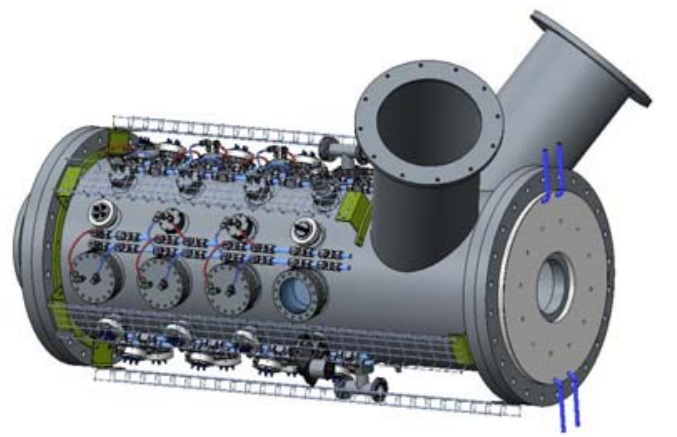

Fig. 5. PMI chamber with diagnostic ports, water services, and cable trays.

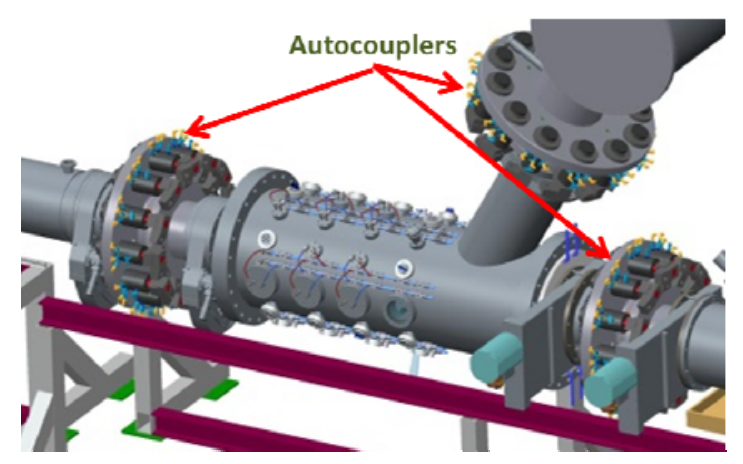

Fig. 6. Autocouplers attached to PMI chamber.

\subsection{Water cooling system}

Because MPEX will operate in steady-state, it is necessary that every plasma facing component and system be water cooled. The total cooling power required to support MPEX, if all systems are operating simultaneously, is estimated to be approximately 2MW, with a flow rate of 670 gallons per minute. The primary vacuum boundaries - the dump tank, the heating and transport regions, the ECH box, and the PMI chamber are to be made of stainless steel, with outer cooling jackets welded on. These are expected to experience heat fluxes of no higher than $10 \mathrm{~kW} / \mathrm{m}^{2}$, with the exception of the PMI chamber and dump tank, which are estimated to experience heat fluxes of $25 \mathrm{~kW} / \mathrm{m}^{2}$. Diagnostics ports, port covers, and heads of larger chambers will be cooled with cooling traces. MPEX will include a few in-vessel components. Skimmers are intended to prevent neutral transport, and because of their close proximity to the plasma, they are expected to experience heat fluxes up to $250 \mathrm{~kW} / \mathrm{m}^{2}$. A limiter will be placed just downstream of the helicon. Because this component will interact with the plasma, it is estimated to experience average heat fluxes of $1 \mathrm{MW} / \mathrm{m}^{2}$. The dump tank will have a plate that is intended to intercept the plasma, experiencing an average flux of $400 \mathrm{~kW} / \mathrm{m}^{2}$. These components will be made of copper (for cooling) bonded to tungsten (to interact with the plasma). The target itself will be designed to handle steady-state heat fluxes of over $10 \mathrm{MW} / \mathrm{m}^{2}$. In addition to these 
components, the helicon and window, the vacuum pipes, roots pumps, gyrotron, power supplies, and the RF transmitter must be water cooled. Three secondary loops are proposed for the MPEX cooling system:

- A high temperature, low pressure loop for vacuum tanks.

- A high temperature, high pressure loop for higher heat flux components.

- A low temperature loop for roots pumps and power supplies.

\section{MPEX target}

The MPEX target system consists of a target, a target puck, and a target holder. Each of these components is discussed below.

\subsection{Target design}

The target for MPEX is a frustum of a cone with a base diameter of $12 \mathrm{~mm}$, top diameter of $10 \mathrm{~mm}$, and thickness of $1 \mathrm{~mm}$ (see Fig 7). All targets will be fabricated by sintering correctly proportioned powdered tungsten and tungsten alloys into the desired disc shape.

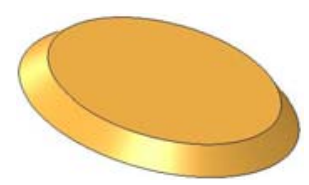

Fig. 7. Target design for MPEX.

Fig. 8 shows the configuration of the target puck and the holder. The target puck holder houses the copper water cooled elements which is in contact with the target puck and the cooling channel in contact with the tungsten shield. The target puck holder is fixed to a telescoping arm for exposing target to plasma in the PMI chamber.

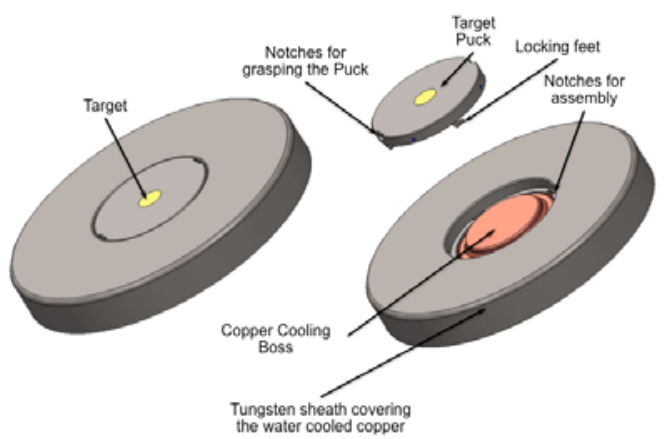

Fig. 8. Target puck and target puck holder assembly.

\subsection{Target puck design}

The target puck houses the target. Since the target has to be periodically replaced, the cooling channels have to be contained inside a target puck holder copper boss and the target puck interfaces with this boss. The target puck consists of the target, grafoil, tungsten retainer and the target securing base (Fig. 9). The grafoil compresses to ensure good contact between the target and the tungsten retainer, which is in contact with the water cooled copper boss. The thickness of the grafoil and the tungsten retainer affects the temperature of the target. The plasma flux on the tungsten was modeled as ringed regions with differing heat fluxes. Integrating the Gaussian distribution of the plasma flux, these regions closely approximated the plasma heat flux on the tungsten target and the target puck holder.

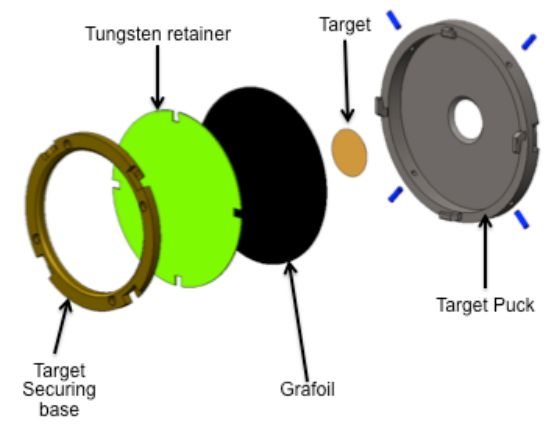

Fig. 9. Target puck assembly.

An aim of the target design is to maintain a uniform temperature on the target. The temperature of the target center can be adjusted by choosing different Grafoil thicknesses. For a given heat flux of $10 \mathrm{MW} / \mathrm{m}^{2}$, the surface temperature can vary between $800{ }^{\circ} \mathrm{C}$ and 1200 ${ }^{\circ} \mathrm{C}$, for example. The cooling of the target has been designed to minimize temperature variations across the target $( \pm 5 \mathrm{~mm})$ to below $3 \%$. The peak target temperature is a function of the thermal resistance of the various layers that make up target puck. It was found through simulation that a thickness of $0.38 \mathrm{~mm}$ for tungsten retainer and $0.5 \mathrm{~mm}$ for Grafoil results in the center tungsten target temperature of $1200^{\circ} \mathrm{C}$. This also results in the copper remaining below its melt temperature, and only $35^{\circ} \mathrm{C}$ rise in water temperature.

\subsection{Target puck holder}

The assembled target puck holder is installed on the target manipulator in the Target Exchange Chamber (TEC) through a telescoping arm. The cooling lines reach the copper base of the target puck holder. The puck holder has a diameter of $12 \mathrm{~cm}$, which is larger than the diameter of the MPEX plasma beam of $10 \mathrm{~cm}$ thus reducing the power load on peripheral structures. The target puck holder has an outer skin layer of tungsten (with a thickness of $1.0 \mathrm{~mm}$ ) with a copper base structure that houses the cooling water lines (Fig. 10). Two target holder designs, $90^{\circ}$ and $15^{\circ}$, are shown. The removable target puck design is same for the $15^{\circ}$ and the $90^{\circ}$ target, allowing them to be used interchangeably. 


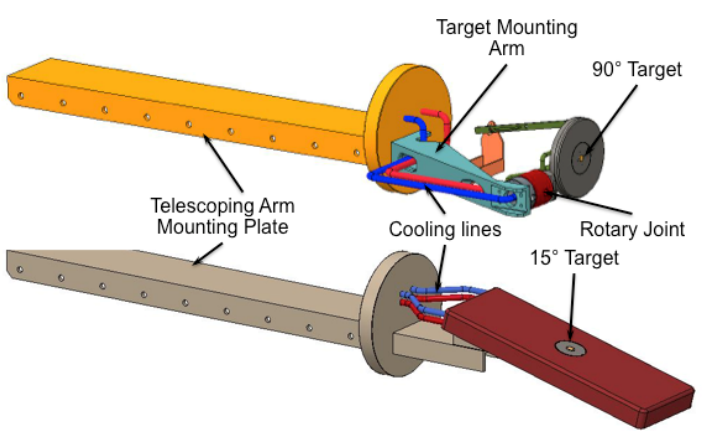

Fig. 10. Target puck holder.

\subsection{Target exchange chamber}

The target exchange chamber houses the target. It has a telescoping arm to extend the target into the PMI chamber. Fig. 11 shows the target exchange chamber connected to the PMI chamber.

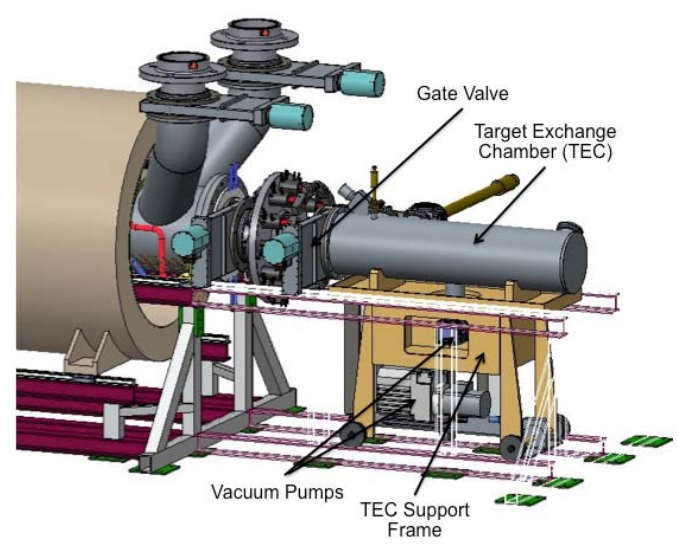

Fig. 11. Target exchange chamber.

A three stage telescoping system made of 316 stainless steel sliders and ceramic wheels (which are vacuum compatible) provides the linear extension for the target arm (Fig. 12). The actuator for this linear slide will be a pneumatic motor to allow it to operate in close proximity to magnetic field. Also, to extend the arm, a push pull chain is used. The push pull chain uses stainless steel with aluminum bronze sprocket to avoid galling between moving parts in a vacuum environment. A vacuum rotary joint is used to couple the vacuum motor to the push-pull chain drive. An extension of 1.6 meters is achieved by the telescoping slide.

Since the target is cantilevered at the end of the arm, there is the possibility that the target will vibrate. To minimize this vibration, the target slides on to a ramp fixed in the PMI chamber when it is extended. The ramp also provides a travel stop for the target to ensure that it is repeatedly positioned at the same location in the PMI chamber (Fig. 13).

The TEC is equipped with a roughing pump and a high vacuum turbo pump. This ensures that the TEC is brought down to $10^{-7}$ mbar pressure as soon as it is isolated from the PMI chamber. The TEC chamber is on rails so that it can be moved to an area that is magnetically isolated from the superconducting magnets. This are will house diagnostic equipment that is sensitive to magnetic field.

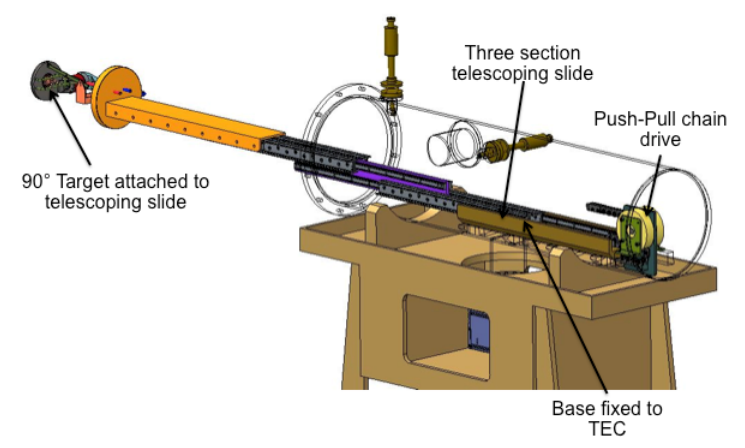

Fig. 12. Telescoping slide.

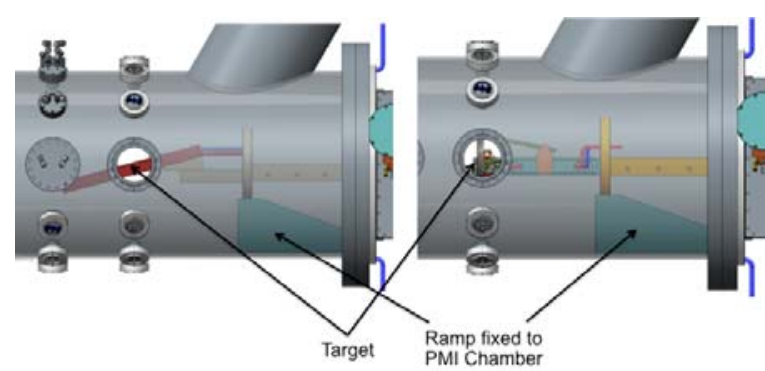

Fig. 13. PMI chamber target views.

\section{References}

[1] J. Reiser, and M. Rieth, Optimization and limitations of known DEMO divertor concepts, Fusion Eng Des, 87(56), (2012), 718-721.

[2] A.R. Raffray, et al, High heat flux components readiness to proceed from near term fusion systems to power plants, Fusion Eng Des, 85, (2010), 93-108.

[3] D. Maisonnier, et al, DEMO and fusion power plan conceptual studies in Europe, Fusion Eng Des, 81, (2006), 1123-1130.

[4] R. Behrisch, et al, Material erosion at the vessel walls of future fusion devices, J Nucl Mater, 313-316 (2003) 388392.

[5] J. Rapp, et al, Construction of the plasma-wall experiment Magnum-PSI, Fusion Eng Des, 85(7-9), (2010), 1455-1459.

[6] J. Rapp, et al, The development of plasma-material interaction facilities for the future of fusion technology, Fusion Sci Technol, 64(2), (2013), 237-244.

[7] J. Rapp et al., Transport simulations of linear plasma generators with the B2.5-Eirene and EMC3-Eirene codes, J. Nucl. Mater. 463 (2015) 510-514. 